\title{
SHORT REPORT \\ The role of symptomatic presentation in influenza A transmission risk
}

\author{
R. WARDELL ${ }^{1}$, K. PREM${ }^{2}$, B. J. COWLING ${ }^{3}$ AND A. R. COOK ${ }^{2,4,5 *}$ \\ ${ }^{1}$ Global Health Division, Research School of Population Health, College of Medicine, Biology and the \\ Environment, The Australian National University, Canberra, Australia \\ ${ }^{2}$ Saw Swee Hock School of Public Health, National University of Singapore and National University Health \\ System, Singapore \\ ${ }^{3}$ WHO Collaborating Centre for Infectious Disease Epidemiology and Control, School of Public Health, Li Ka \\ Shing Faculty of Medicine, The University of Hong Kong, Hong Kong Special Administrative Region, China \\ ${ }^{4}$ Program in Health Services and Systems Research, Duke-NUS Medical School, Singapore \\ ${ }^{5}$ Department of Statistics and Applied Probability, National University of Singapore, Singapore
}

Received 23 March 2016; Final revision 24 September 2016; Accepted 31 October 2016; first published online 5 December 2016

\section{SUMMARY}

Computer models can be useful in planning interventions against novel strains of influenza.

However such models sometimes make unsubstantiated assumptions about the relative infectivity of asymptomatic and symptomatic cases, or conversely assume there is no impact at all. Using household-level data from known-index studies of virologically confirmed influenza A infection, the relationship between an individual's infectiousness and their symptoms was quantified using a discrete-generation transmission model and Bayesian Markov chain Monte Carlo methods. It was found that the presence of particular respiratory symptoms in an index case does not influence transmission probabilities, with the exception of child-to-child transmission where the donor has phlegm or a phlegmy cough.

Key words: Bayesian statistics, influenza A, modelling, symptoms, transmission.

\section{INTRODUCTION}

The emergence of several novel strains of influenza and respiratory viruses over the past decade has challenged public health systems [1,2]. Computational models can be useful tools when planning for epidemics, especially those caused by novel pathogens for which there is little evidence for the effectiveness of interventions. Examples where models' predictions were subsequently validated empirically include the effectiveness of antiviral ring prophylaxis $[3,4]$ and

\footnotetext{
* Author for correspondence: Dr A. R. Cook, Saw Swee Hock School of Public Health, Tahir Foundation Building, 12 Science Drive 2, Singapore 117549. Email: alex.richard.cook@gmail.com
}

the closure of schools during influenza pandemics to slow down the spread of infection [5, 6]. However, models are inherently limited by the accuracy of the data or assumptions used to construct them, and there are key unknowns that may impact the reliability of findings derived from modelling studies. For interventions against influenza pandemics, for instance, models need to make assumptions about the relative infectivity of asymptomatic or subclinical cases these individuals are less likely to be detected by surveillance or to reduce their social contacts, and so may play an important role in propagating transmission. Many of the most influential models [7, 8] assume that asymptomatic cases are half as infectious as symptomatic ones, an assumption that dates back 
to Elveback et al.'s self-declared 'guesstimate' in the 1970s [9], but which does not appear to have been substantiated by evidence then or since.

This study quantifies the relationship between an individual's infectiousness and their symptoms, using household-level data in which index cases and their cohabitants had influenza A infection virologically confirmed and logged symptoms in a diary, and a discrete-generation transmission model combined with Bayesian Markov chain Monte Carlo methods.

\section{METHODS}

\section{Data}

Data were obtained from household influenza studies conducted in Hong Kong between February 2007 and June 2009. These were from a community-based randomized controlled trial that recruited individuals with influenza-like illness from outpatient clinics across Hong Kong, and their households. Detailed information on the study design can be found in Cowling et al. [10]. Briefly, index cases were recruited into the study if they (i) experienced the onset of at least two symptoms of acute respiratory illness (temperature $\geqslant 37.8^{\circ} \mathrm{C}$, headache, myalgia or cough) within $48 \mathrm{~h}$, (ii) lived in a household with at least two individuals, none of whom had a reported acute respiratory illness in the previous 14 days, and (iii) had a positive result using the QuickVue Influenza A + B rapid diagnostic test (Quidel, USA). At a later date, influenza was confirmed using either RTPCR (during the 2008/2009 seasons) or viral culture (during 2007). The study involved three home visits over a period of $7-10$ days. The first home visit occurred ideally within $36 \mathrm{~h}$ of recruitment, during which nasal and throat specimens were collected from all consenting household members. Recruited individuals and household members were then asked to keep a self-reported daily symptom diary for about 1 week. Subsequent home visits occurred 3 and 6 days after recruitment, to collect additional nasal and throat specimens from household members. All specimens were tested by RT-PCR (during the 2008/2009 seasons) or viral culture (during 2007).

All index cases with laboratory-confirmed influenza A (either RT-PCR or culture) were identified. For each of these individuals and their household members, we extracted from their symptom diaries the presence of the following respiratory symptoms: sore throat, cough, runny nose, phlegm, or the combination of cough and phlegm; missing values ( $\sim 1.5 \%$ of symptoms) were treated as indicating symptom absence. Household members were considered to display evidence of infection if they had laboratory-confirmed influenza A during follow-up as the accuracy of these tests is high ( $\sim 90 \%$ sensitivity, $\sim 80 \%$ specificity [11]). Individuals were categorized as being adults if aged $>18$ years. The dataset used in the models has 462 influenza A cases (331 index cases, 131 secondary cases).

\section{Model}

We developed an age-structured chain binomial model [12] with transmission probabilities that varied by symptom presence. This model was separately fit to each symptom one at a time. This represents transmission as occurring in discrete infection generations, with those infected on generation $g$ able to infect those as yet uninfected on generation $g+1$ but not on subsequent generations. The transmission probability from individual $i$ of age group $a_{i}$ ( $=0$ if $i$ is a child and 1 otherwise), where $i$ has symptoms if $s_{i}=1$ and not if 0 , to individual $j$ is $\pi_{i j}=p_{a_{i}, a_{j}, s_{i}}$. These eight risks were modelled as free parameters.

Infection of those uninfected within the household at each generation is assumed to be independent, with risk from multiple sources compounding, i.e. if $\mathcal{I}_{g}$ is the set of infection in generation $g$, infection to $j \notin \mathcal{I}_{g}$ is with probability $1-\prod_{i \in \mathcal{I}_{g}}\left(1-\pi_{i j}\right)$. Transmission ends when no new infections occur in any generation.

Four variants of the basic model were considered no symptoms, half infectious, free parameter and multiple symptoms. The first ignored symptoms to obtain baseline transmission risks by age group of infective and household member. The second fixed the infectivity of asymptomatic cases at half that of symptomatic cases (as in Elveback et al. [9] and more recent papers). The free parameter model by contrast has an additional class of parameters that accounts for any respiratory symptoms present. The multiple symptoms model used the number $n_{i}$ of respiratory symptoms for each case $i$ with infection risk $\pi_{i j}=\alpha_{a_{i}, a_{j}}+n_{i} \beta_{a_{i}, a_{j}}$, truncated to $[0,1]$ and the probability of infection varies with the number of symptoms.

For all models, the likelihood function could be evaluated directly if the generation of each infection were known. This happens only if the index fulfils case criteria, or if the index and one cohabitant do. 
For more infections, the order of infection must be explored, either by summing the probabilities of all infection generation combinations, or by data augmentation [13], i.e. treating the unobserved generation as an additional parameter to estimate (further details available in the Supplementary material). As there were households with four infections, we used the latter, as it was more computationally efficient than the former when the number of combinations was large.

Flat priors over $[0,1]$ for all proportions were taken (or on $[-10,10]$ for the parameters of the multisymptom model) and a Markov chain Monte Carlo algorithm was used to sample the resulting posterior. This used 10 chains each of 100000 iterations with every 100th iteration retained and convergence assessed by visual inspection of trace plots. Posterior samples were converted to obtain equal tailed $95 \%$ credible intervals (CIs) for relevant estimands, and the distribution of risks with and without symptoms were compared by the posterior distribution of absolute difference in transmission risk. No adjustment for multiple estimation was attempted.

We screened for the model that fits the data best of the candidate models we considered using Deviance Information Criteria (DIC) described by Gelman et al. [14]. All analyses were performed in R statistical environment with a custom-designed script.

\section{RESULTS}

The risk of transmission from an infected child to a second child was $21 \cdot 9 \%$ (95\% CI $14 \cdot 5-30 \cdot 2)$, higher than the corresponding risk to a cohabiting adult [11.4\%, 95\% CI 9.1-14.2; relative risk (RR) 1.9, 95\% CI 1.2-2.8]. Adults were less likely than children to transmit infection to other adults $(6.9 \%, 95 \%$ CI $4 \cdot 4-10 \cdot 1$; RR $0 \cdot 6,95 \%$ CI $0 \cdot 3-0 \cdot 96$ ) or to children $(10 \cdot 0 \%, 95 \%$ CI $4 \cdot 5-18 \cdot 0$; RR $0 \cdot 5,95 \%$ CI $0 \cdot 2-1 \cdot 0)$.

Estimated transmission risks from either adults or children to adults were not strongly affected by any of the respiratory symptoms assessed (Fig. 1, Table 1), which may have modulated transmission potential by at most 8.4 percentage points (from adults) or 13 percentage points (from children). There was much less certainty in the effect of adult symptoms on transmission potential to children (Fig. 1). In contrast, there was clear evidence that the presence of phlegm or a phlegmy cough in children was associated with an increased risk of infection in other children in the household $[20$ percentage points (95\% CI 3.1-35) for phlegm, 21 percentage points

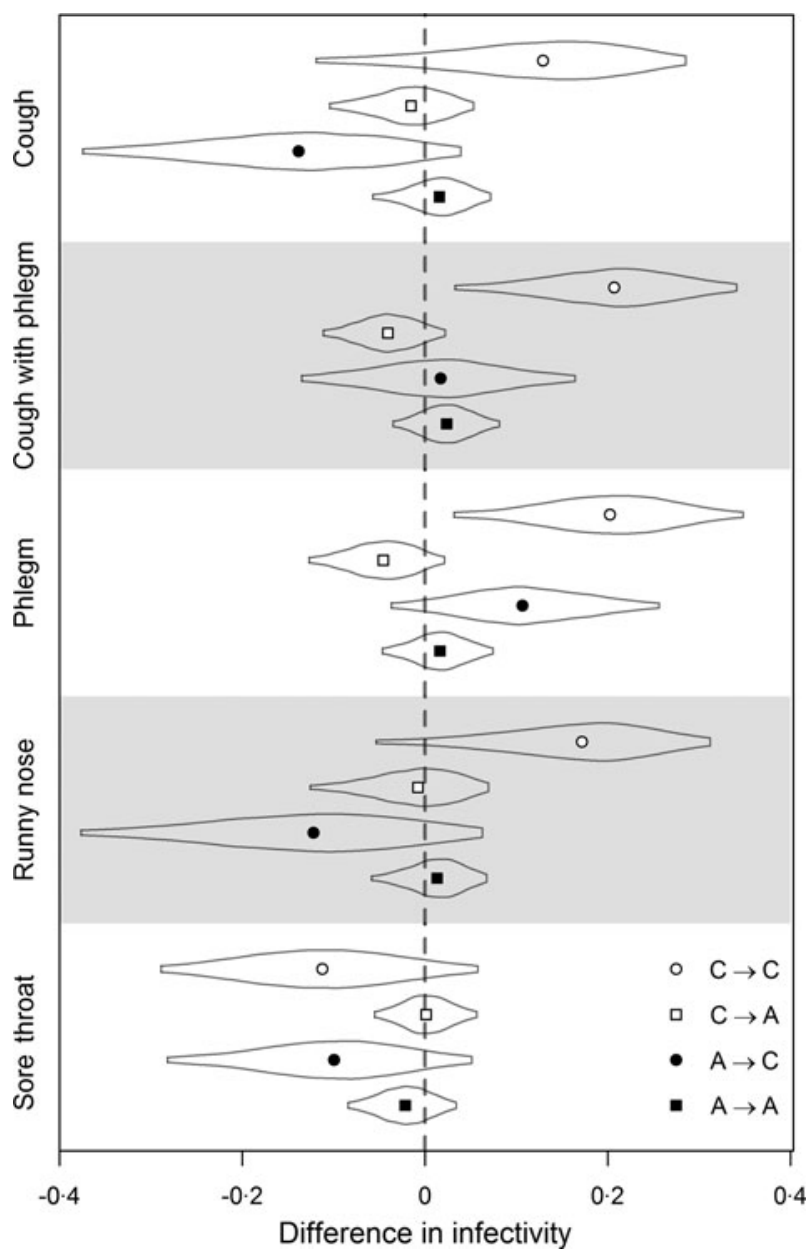

Fig. 1. The effects of respiratory symptoms on the transmission risks from either adults or children to adults or children. Points are posterior medians, curves are posterior distributions truncated to within 95\% credible intervals. Differences are in probability of infection between combinations of ages. C, Children; A, adults.

(95\% CI 3.2-34) for phlegm and cough]. The effect of cough and runny nose was in the same direction, although the sample sizes did not permit confirmation. The presence of a sore throat in infected children did not notably increase transmission risk to other children.

For the model that assessed the effect of total number of symptoms, ignoring their type, the number of symptoms was not associated with infection risk, with non-significant effect sizes ranging from $-0 \cdot 5 \%$ to $0.6 \%$ depending on the age combinations.

There was moderate to strong support in favour of the no-symptom effect model compared to the models that allowed infectivity of those with symptoms to be arbitrarily higher $(\triangle \mathrm{DIC}=6.02)$ or twice $(\Delta \mathrm{DIC}=$ $2.67)$ that of the asymptomatic individuals and to a 
Table 1. Estimated transmission risks from either adults or children to adults or children with or without symptoms

\begin{tabular}{|c|c|c|c|c|c|c|c|}
\hline \multirow[b]{2}{*}{ Symptoms } & \multirow[b]{2}{*}{ Path: } & \multicolumn{2}{|c|}{ Risk without symptoms } & \multicolumn{2}{|c|}{ Risk with symptoms } & \multicolumn{2}{|c|}{ Absolute risk difference } \\
\hline & & Median & $95 \% \mathrm{CI} \dagger$ & Median & $95 \% \mathrm{CI} \dagger$ & Median & $95 \% \mathrm{CI} \dagger$ \\
\hline \multirow[t]{4}{*}{ Cough } & $\mathrm{C} \rightarrow \mathrm{C}$ & $0 \cdot 12$ & $(0 \cdot 0095-0 \cdot 36)$ & $0 \cdot 25$ & $(0 \cdot 16-0 \cdot 36)$ & $0 \cdot 13$ & $(-0 \cdot 12$ to $0 \cdot 29)$ \\
\hline & $\mathrm{C} \rightarrow \mathrm{A}$ & $0 \cdot 12$ & $(0 \cdot 061-0 \cdot 21)$ & $0 \cdot 11$ & $(0 \cdot 08-0 \cdot 14)$ & $-0 \cdot 015$ & $(-0 \cdot 1$ to $0 \cdot 054)$ \\
\hline & $\mathrm{A} \rightarrow \mathrm{C}$ & $0 \cdot 23$ & $(0 \cdot 07-0 \cdot 47)$ & $0 \cdot 091$ & $(0 \cdot 034-0 \cdot 19)$ & $-0 \cdot 14$ & $(-0.38$ to $0 \cdot 041)$ \\
\hline & $\mathrm{A} \rightarrow \mathrm{A}$ & $0 \cdot 06$ & $(0 \cdot 019-0 \cdot 13)$ & $0 \cdot 076$ & $(0 \cdot 045-0 \cdot 12)$ & $0 \cdot 016$ & $(-0.057$ to 0.072$)$ \\
\hline \multirow[t]{4}{*}{ Cough with phlegm } & $\mathrm{C} \rightarrow \mathrm{C}$ & $0 \cdot 071$ & $(0 \cdot 006-0 \cdot 23)$ & $0 \cdot 28$ & $(0 \cdot 19-0 \cdot 4)$ & $0 \cdot 21$ & $(0 \cdot 032$ to $0 \cdot 34)$ \\
\hline & $\mathrm{C} \rightarrow \mathrm{A}$ & $0 \cdot 14$ & $(0 \cdot 087-0 \cdot 2)$ & $0 \cdot 1$ & $(0 \cdot 071-0 \cdot 13)$ & $-0 \cdot 041$ & $(-0.11$ to 0.022$)$ \\
\hline & $\mathrm{A} \rightarrow \mathrm{C}$ & $0 \cdot 12$ & $(0 \cdot 037-0 \cdot 24)$ & $0 \cdot 13$ & $(0 \cdot 045-0 \cdot 27)$ & $0 \cdot 017$ & $(-0 \cdot 14$ to $0 \cdot 17)$ \\
\hline & $\mathrm{A} \rightarrow \mathrm{A}$ & $0 \cdot 058$ & $(0 \cdot 025-0 \cdot 11)$ & $0 \cdot 083$ & $(0 \cdot 046-0 \cdot 13)$ & $0 \cdot 024$ & $(-0.035$ to 0.082$)$ \\
\hline \multirow{4}{*}{ Phlegm } & $\mathrm{C} \rightarrow \mathrm{C}$ & $0 \cdot 065$ & $(0 \cdot 0057-0 \cdot 22)$ & $0 \cdot 28$ & $(0 \cdot 17-0 \cdot 39)$ & $0 \cdot 2$ & $(\mathbf{0 . 0 3 1}$ to $\mathbf{0 \cdot 3 5 )}$ \\
\hline & $\mathrm{C} \rightarrow \mathrm{A}$ & $0 \cdot 15$ & $(0 \cdot 088-0 \cdot 22)$ & $0 \cdot 1$ & $(0 \cdot 073-0 \cdot 13)$ & $-0 \cdot 046$ & $(-0.13$ to 0.022$)$ \\
\hline & $\mathrm{A} \rightarrow \mathrm{C}$ & $0 \cdot 065$ & $(0 \cdot 0091-0 \cdot 18)$ & $0 \cdot 17$ & $(0 \cdot 073-0 \cdot 32)$ & $0 \cdot 11$ & $(-0.038$ to $0 \cdot 26)$ \\
\hline & $\mathrm{A} \rightarrow \mathrm{A}$ & $0 \cdot 061$ & $(0 \cdot 025-0 \cdot 12)$ & $0 \cdot 078$ & $(0 \cdot 043-0 \cdot 12)$ & $0 \cdot 016$ & $(-0.047$ to 0.075$)$ \\
\hline \multirow[t]{4}{*}{ Runny nose } & $\mathrm{C} \rightarrow \mathrm{C}$ & $0 \cdot 076$ & $(0 \cdot 003-0 \cdot 29)$ & $0 \cdot 25$ & $(0 \cdot 16-0 \cdot 36)$ & $0 \cdot 17$ & $(-0.054$ to 0.31$)$ \\
\hline & $\mathrm{C} \rightarrow \mathrm{A}$ & $0 \cdot 12$ & $(0 \cdot 047-0 \cdot 23)$ & $0 \cdot 11$ & $(0 \cdot 083-0 \cdot 14)$ & $-0 \cdot 0078$ & $(-0.13$ to 0.07$)$ \\
\hline & $\mathrm{A} \rightarrow \mathrm{C}$ & $0 \cdot 23$ & $(0 \cdot 052-0 \cdot 46)$ & $0 \cdot 1$ & $(0 \cdot 04-0 \cdot 19)$ & $-0 \cdot 12$ & $(-0.38$ to 0.064$)$ \\
\hline & $\mathrm{A} \rightarrow \mathrm{A}$ & $0 \cdot 061$ & $(0 \cdot 019-0 \cdot 13)$ & $0 \cdot 074$ & $(0 \cdot 044-0 \cdot 11)$ & $0 \cdot 013$ & $(-0.059$ to 0.068$)$ \\
\hline \multirow[t]{4}{*}{ Sore throat } & $\mathrm{C} \rightarrow \mathrm{C}$ & $0 \cdot 3$ & $(0 \cdot 17-0 \cdot 45)$ & $0 \cdot 18$ & $(0 \cdot 088-0 \cdot 3)$ & $-0 \cdot 11$ & $(-0.29$ to 0.059$)$ \\
\hline & $\mathrm{C} \rightarrow \mathrm{A}$ & $0 \cdot 11$ & $(0 \cdot 071-0 \cdot 16)$ & $0 \cdot 11$ & $(0 \cdot 079-0 \cdot 15)$ & $0 \cdot 0013$ & $(-0.055$ to 0.057$)$ \\
\hline & $\mathrm{A} \rightarrow \mathrm{C}$ & $0 \cdot 18$ & $(0 \cdot 065-0 \cdot 36)$ & $0 \cdot 081$ & $(0 \cdot 021-0 \cdot 19)$ & $-0 \cdot 1$ & $(-0.28$ to 0.053$)$ \\
\hline & $\mathrm{A} \rightarrow \mathrm{A}$ & $0 \cdot 085$ & $(0 \cdot 043-0 \cdot 14)$ & $0 \cdot 062$ & $(0 \cdot 033-0 \cdot 11)$ & -0.022 & $(-0.084$ to 0.035$)$ \\
\hline
\end{tabular}

CI, Credible interval; C, children, A, adults.

$\dagger$ Posterior medians of the estimated transmission probabilities with corresponding $95 \%$ credible intervals (CI).

$\$$ The arrows indicate the transmission pathways of influenza $\mathrm{A}$ with the donor on the left and the inheritor on the right; e.g. $\mathrm{C} \rightarrow \mathrm{A}$ represents a child-to-adult transmission.

model that considered the presence of multiple symptoms $(\Delta \mathrm{DIC}=57.57)$.

\section{DISCUSSION}

Our results suggest that the presence of particular respiratory symptoms in influenza-infected individuals does not influence transmission probabilities, unless there is child-to-child transmission. Additionally, the number of symptoms present does not influence transmission probabilities, whether treated linearly or dichotomized (not shown). Taken together these results suggest that the absence of any particular symptoms in an influenza case, or the overall number of symptoms, might not be associated with a decreased risk of infectivity. This is an important finding, as several influential influenza A models assume that symptomatic cases are twice as infectious as asymptomatic cases [7, 8].

Our results, however, reveal that the presence of specific respiratory symptoms, notably phlegm or a cough with phlegm, in paediatric index cases increases their risk of transmitting influenza A to other children. The occurrence of this phenomenon may be explained by studies finding that children are more likely to spread infection to other household members, as well as the notion that children are more at risk of influenza A infection due to their increased susceptibility and higher rate of contact with potentially contaminated surfaces $[15,16]$. It is possible that a more sophisticated analysis that pooled information between symptoms might illuminate the effect of other symptom presentations, but the lack of statistical significance for the models combining symptoms suggests otherwise, unless the sample size were substantially larger.

There are several assumptions underlying our study. Due to the study design we were unable to correct for pre-season antibody levels, or for the possibility that an asymptomatic case preceded the assumed index. However, other studies have shown that even after correcting for preseason HI titres, children have an elevated risk of household infection compared to adults [17], and the impact of prior immunity or cryptic indices is likely to impact the risk of infection but not the difference due to symptom presentation. We assumed that households are independent of each other, which is plausible in the absence of spatial clustering of recruited household, and that all secondary household cases obtained influenza from the index case. 
The method of recruitment into this study has inherent selection bias, favouring index cases that have severe enough symptoms to seek medical assistance from their healthcare provider, and high enough viral loads to have a laboratory-confirmed case of influenza A. Index cases were recruited if they experienced the onset of at least two symptoms of acute respiratory illness, so the effect of any one symptom could be confounded by the presence of the other symptom whose presence co-determines enrolment eligibility. Despite these limitations, this study suggests that assumptions in modelling papers about the rate of asymptomatic transmission may need to be reviewed.

\section{SUPPLEMENTARY MATERIAL}

For supplementary material accompanying this paper visit https://doi.org/10.1017/S0950268816002740.

\section{ACKNOWLEDGEMENTS}

R.W. was supported by a New Colombo Plan scholarship from the Australian Department of Foreign Affairs and Trade (DFAT). K.P. and A.R.C. were supported by funding from the Ministry of Education, Ministry of Health, Ministry of Defence, and the National University Health System, all Singapore (grant nos. CDPHRG/ 0009/2014, NUHSR0/2013/142IH7N9104, PROJECT MODUS 9014100379). B.J.C. was supported by the National Institute of General Medical Sciences (grant U54 GM088558), a grant from the Research Grants Council of the Hong Kong Special Administrative Region, China (Project No. T11-705/14N), and a commissioned grant from the Health and Medical Research Fund from the Government of the Hong Kong Special Administrative Region. The original household trial in Hong Kong was supported by the United States Centers for Disease Control and Prevention (grant no. 1 U01 CI000439). The funders had no role in study design, data collection and analysis, decision to publish, or preparation of the manuscript.

\section{DECLARATION OF INTEREST}

B.J.C. reports receipt of research funding from Sanofi Pasteur and MedImmune Inc. for studies of influenza vaccination effectiveness.

\section{REFERENCES}

1. Briand S, Mounts A, Chamberland M. Challenges of global surveillance during an influenza pandemic. Public Health 2011; 125: 247-256.

2. Liu Y. China's public health-care system: facing the challenges. Bulletin of the World Health Organization 2004; 82: 532-538.

3. Ferguson NM, et al. Strategies for containing an emerging influenza pandemic in Southeast Asia. Nature 2005; 437: 209-214.

4. Lee VJ, et al. Oseltamivir ring prophylaxis for containment of 2009 H1N1 influenza outbreaks. New England Journal of Medicine 2010; 362: 2166-2174.

5. Cauchemez S, et al. Estimating the impact of school closure on influenza transmission from Sentinel data. Nature 2008; 452: 750-754.

6. Wu JT, et al. School closure and mitigation of pandemic (H1N1) 2009, Hong Kong. Emerging Infectious Diseases 2010; 16: 538-541.

7. Chao DL, et al. FluTE, a publicly available stochastic influenza epidemic simulation model. PLoS Computational Biology 2010; 6: e1000656.

8. Halloran ME, et al. Modeling targeted layered containment of an influenza pandemic in the United States. Proceedings of the National Academy of Sciences USA 2008; 105: 4639-4644.

9. Elveback LR, et al. An influenza simulation model for immunization studies. American Journal of Epidemiology 1976; 103: 152-165.

10. Cowling BJ, et al. Facemasks and hand hygiene to prevent influenza transmission in households: a cluster randomized trial. Annals of Internal Medicine 2009; 151: 437.

11. Zambon M, et al. Diagnosis of influenza in the community: relationship of clinical diagnosis to confirmed virological, serologic, or molecular detection of influenza. Archives of Internal Medicine 2001; 161: 2116.

12. Bailey NT. The Mathematical Theory of Infectious Diseases, 2nd edn. London: Hafner Press/Macmillan Publishing Co., 1975.

13. Gibson G, et al. Estimating parameters in stochastic compartmental models using Markov chain methods. Mathematical Medicine and Biology 1998; 15: 19-40.

14. Gelman A. Bayesian Data Analysis, 2nd edn. Boca Raton, FL: Chapman \& Hall/CRC, 2004.

15. Viboud C, et al. Risk factors of influenza transmission in households. British Journal of General Practice 2004; 54: 684-689.

16. Monto AS. Studies of the community and family: acute respiratory illness and infection. Epidemiologic Reviews 1994; 16: 351-373.

17. Cauchemez S, et al. Determinants of influenza transmission in South East Asia: insights from a household cohort study in Vietnam. PLoS Pathogens 2014; 10: e1004310. 